\title{
Solar Energetic Particles in SOHO/EIT images: cleaning images and particle diagnostics
}

\author{
Victor V. Grechnev \\ Institute of Solar Terrestrial Physics, Irkutsk, Russia, email: grechnev@iszf.irk.ru
}

Solar energetic particles generated in solar proton events produce in SOHO/EIT (and LASCO) images well-known 'snowstorm' effect which interferes analyses of those images. Similar 'snowstorms' are also observed in TRACE and CORONAS-F/SPIRIT images due to particles continuously present in the radiation belts and polar caps.

Assuming the particle flux to be random, uniform, and stationary, one can expect the following properties of the 'snowstorm': (1) the positions of the 'snowflakes' in different images are independent; (2) their density in an image is proportional (a) to the particle flux, and (b) to the exposure time. These properties give us a possibility to suppress the snowstorm using other images from the same series and put limitations for that. Depending on the particular task to be solved and the data set available, one can choose different techniques. Of course, there are restrictions, and if we have a frame uniformly white because of complete coverage by the snowstorm, then nothing can be revealed.

We propose the following methods to clean images: (1) replacement of each pixel in an image with its minimum value from adjacent frames; in studies of dimmings(2) displaying only darkening regions and (3) composing a single image as the minimum value over the whole set of difference images (dimming 'portrait' of an event); (4) full restoration through the replacement of each pixel within 'snowflakes' with its minimum value from adjacent images, with the snowflakes found in the current image as drastic brightenings with respect to adjacent images.

From the comparison of the snowstorm density time profiles with fluxes of energetic protons and electrons recorded with GOES monitors during two solar proton events (2000/07/14 and 2003/10/28 - figure 1) it follows that snowstorms in SOHO/EIT (and LASCO) images are mainly due to protons with energies of order $40-80 \mathrm{MeV}$, and/or secondary particles produced by those protons (correlation $>0.95$ ).

Currently, three kinds/domains of acceleration are discussed: (1) on the Sun, (2) by shockwave fronts moving in the interplanetary space from the Sun to the Earth, (3) by electromagnetic fields in the solar corona and the interplanetary space. If there would be an opportunity to perform the relative timing of protons and ultra-relativistic electrons in different energy bands separately, it could give some light to the localization of the acceleration sites and the acceleration mechanisms. Our study shows that useful byproduct of imaging data can be directly related to particle acceleration problems, and this could be taken into account in forthcoming space missions which would allow recording particle fluxes from very different points in the heliosphere.

\section{Acknowledgements}

The author thanks I. Chertok, A. Golovko, O. Bugaenko, A. Uralov, V. Dvornikov, and V. Sdobnov for fruitful discussions. We thank the instrumental teams operating GOES and $\mathrm{SOHO}$ spacecrafts and instruments onboard them. SOHO is a project of international 


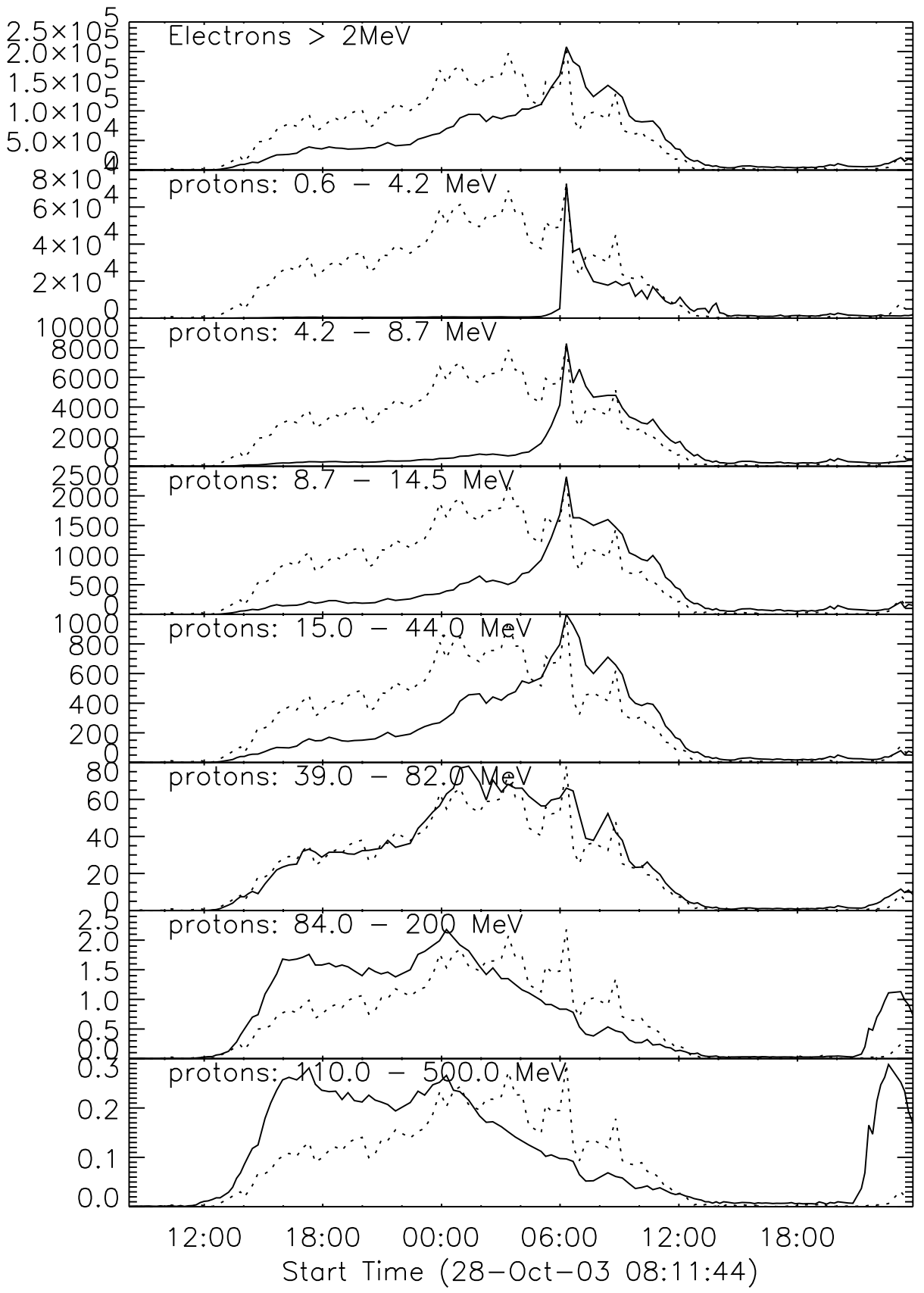

Figure 1. Comparison of particle fluxes with the density of the 'snowstorm' in SOHO/EIT images (dotted) for the event of October 28, 2003

cooperation between ESA and NASA. This work is supported by the Russian RFBR grants 03-02-16591, 03-07-90054, and the Ministry of Education and Science grant 447.2003 .2 . 